\title{
ARTIGO
}

\section{Necesidad de fortalecimiento de la extensión universitaria como componente del proyecto académico, con miras a la evaluación y acreditación}

\author{
The need to strengthen the college extension \\ as a component of the academic project, \\ aiming at evaluation and accreditation
}

Margarita Escobar de MOREL $^{1}$

\section{RE S U M E N}

La efectividad de la extensión universitaria como uno de los pilares de la educación superior sólo puede lograrse mediante el mejoramiento de la calidad de sus resultados y su impacto en los grupos con los cuales se relaciona. La evaluación representa una de las estrategias que pueden contribuir a ese mejoramiento; por ello, se propone un esquema de auto-evaluación institucional y, particularmente, se detallan los criterios de evaluación de la extensión.

Palabras clave: educación superior, extensión universitaria, evaluación.

A B S T R A C T

The effectiveness of the college extension programs as pillars of High Education can only be achieved through the improvement of their results and impact on the

\footnotetext{
1 Profesora Licenciada. Profesora Titular en la Carrera de Bibliotecología de la Facultad Politécnica de la Universidad Nacional de Asunción. E-mail: info@cideb.org.py

Recebido e aceito para publicação em 8/7/2003.
} 
groups they are meant for. Evaluation is one of the strategies that may contribute to such improvements; thus, this paper proposes a plan for institutional self evaluation and, mainly, specifies the criteria to be used in evaluating the extension practices.

Key words: High Education, college extension, evaluation.

\section{N TRO D U C IÓ N}

En nuestra región tienden a coincidir las preocupaciones desde los distintos estamentos y actores del proceso educativo con relación a lo que deberían ser los objetivos y demandas de la educación.

Bello de Arellano (1998) sostiene que los problemas educativos son vistos como una de las consecuencias del agotamiento de las posibilidades de los estilos tradicionales de enseñanza y gestión educativas. Ante esto existe una perentoria necesidad de propiciar cambios profundos en las estructuras educativas, que permitan articular de manera efectiva las demandas económicas, sociales, políticas y culturales, con los procesos de formación y educación.

El punto focal de una reforma educativa debe ser la elevación significativa y generalizada de la calidad de la educación, porque ahí está ahora su principal problema y porque en esa dirección se irán articulando las necesidades y demandas de la sociedad.

Un aspecto que ha caracterizado a la Universidad Nacional de Asunción por muchos años ha sido la tendencia a priorizar la formación profesional y descuidando su vinculación con la sociedad, en el marco del desarrollo general del país.

El los últimos años, las exigencias de calidad impuestas a la educación superior a nivel regional, propiciaron la práctica de la evaluación para la acreditación de programas académicos. Las pautas y criterios establecidos para ello dan un significado similar en importancia a lo académico, a lo científico y a la vinculación con la sociedad. De ahí que últimamente se hayan iniciado acciones tendientes a desarrollar esos sectores.

El desarrollo del pensamiento científico es un rasgo necesario para la educación superior, la que no puede considerarse como tal si no se desarrolla en el estudiante el pensamiento reflexivo, científico y crítico. Específicamente en los niveles de postgrado es donde se debe producir nuevos conocimientos a través de la investigación.

En las instituciones educativas superiores, el valor de la actividad científica se destaca en las tres funciones básicas de docencia, extensión e investigación, pues solamente a través de la investigación se logra que las otras dos funciones se realicen en una forma dinámica, sistemática y formulada en conocimientos objetivos, reales y actualizados. Se requiere por lo tanto, que los profesores estén preparados, capacitados y actualizados, para llevar adelante con éxito las actividades en las que se manifieste la vinculación mencionada.

En el relacionamiento entre la teoría y la práctica es que el alumno puede observar, analizar, criticar y buscar información sobre los problemas de la realidad, a fin de escoger alternativas, presentar y ejecutar soluciones basadas en el análisis, contribuyendo a modificar situaciones del ambiente social del cual es parte.

En el proceso de análisis de los problemas y la búsqueda de respuesta a los mismos dentro de un contexto social, cultural, económico y político, se requiere un tratamiento interdisciplinario, lo cual deberá ser promovido y desarrollado en el estudiante a través de toda su formación. 
Sin embargo se observa un énfasis desproporcionado en el ejercicio de las funciones que corresponden a los componentes académico, científico y extensional en las universidades de nuestro país. La investigación y la extensión universitaria generalmente no están incorporados al diseño curricular y al priorizarse el componente académico, se descuida el desarrollo en los estudiantes del pensamiento científico y de una sensibilidad hacia lo social: el resultado, salvo honrosas excepciones, tesis mediocres en su gran mayoría y ausencia de la universidad (institucionalmente) en los grandes debates nacionales y contemporáneos.

Todo esto se plantea por el incumplimiento de lo establecido como políticas y estrategias en la normativa de la educación superior, que plantea la formación integral del individuo, con el desarrollo de las actividades académicas, científicas y de servicio social.

De persistir esta situación se seguirían formando personas sólo con conocimientos técnico-profesionales pero con escasas habilidades y capacidades para responder creativamente a las situaciones cambiantes de la sociedad.

La relación que se establece entre estos tres componentes depende de la concepción que tienen las instituciones educativas sobre su papel en la sociedad, su compromiso y su conocimiento de la realidad social.

Una reciente investigación sobre la extensión en 21 instituciones de educación superior de Asunción y San Lorenzo revela:

- Sólo 11 instituciones cuentan en su estructura organizativa con un sector o departamento dedicado específicamente a la extensión.

- Sólo 7 instituciones tienen definidos los objetivos y funciones del área de extensión.

- Se involucran en las actividades de extensión los alumnos, profesores, funcionarios y directivos.
- Entre las principales dificultades para realizar las actividades de extensión se mencionan el tiempo disponible (universidades privadas) y los recursos económicos (Universidad Nacional).

- Las actividades de extensión que las universidades privadas realizan con mayor regularidad son las pasantías, convenios interinstitucionales, promoción de ofertas educativas, cursos extracurriculares y viajes de estudio.

- Las actividades de extensión que la Universidad Nacional (a través de sus 15 unidades académicas) realiza con mayor regularidad son los cursos extracurriculares, los viajes de estudio, servicios asistenciales a la comunidad, servicios profesionales a empresas, pasantías, asesorías y consultorías.

- Los beneficios mencionados con respecto a:

- la comunidad: desarrollo social y mejor calidad de vida;

- los estudiantes: desarrollo profesional y afianzamiento de conocimientos;

- la institución: pertinencia curricular y logro de los fines.

Estos datos permiten conocer mejor la actual situación de la extensión universitaria en Paraguay, dejando entrever las debilidades detectadas de manera a encaminar acciones tendientes a fortalecer el sector.

Es oportuno mencionar a Femoso (1996) por destacar que las misiones que una universidad tiene hoy son mucho más complejas y variadas que las que la sociedad le encomendaba en épocas pasadas. Actualmente la universidad debe enseñar y debe hacer investigación y ofrecer servicios que den respuestas a problemas concretos que tengan las empresas o los sectores públicos. Además, deberá colaborar en la formación continuada de los profesionales que ya dejaron las aulas, atenta 
a la demanda creciente de la educación de adultos.

A raíz de todas estas actividades es que se viene prestando cada vez mayor atención a la calidad que deben reunir las instituciones de educación superior. Van Vught, citado por Femoso (1996) afirma en este sentido que las universidades tienen que hacer frente cada vez más a la necesidad de mostrar a la sociedad su pertinencia, calidad y responsabilidad.

Malo (1996) destaca que todo lo dicho anteriormente es reflejo de sociedades cambiantes, complejas, que exigen cosas distintas de sus universidades e instituciones de educación superior. Hoy las sociedades esperan que dichas instituciones de educación superior les generen los cuadros humanos calificados que requieren sus mercados de trabajo; las provean de los estudiosos y practicantes de las letras y las artes; les preparen los empresarios y profesionales innovadores que estimulen las transformaciones sociales; les preparen y doten de los científicos y tecnólogos que las coloque o mantenga en situación competitiva; les proporcionen los individuos que analicen y den respuesta a los múltiples y sofisticados problemas que les aquejan; y que formen a los académicos y maestros que pueblen sus diversas instituciones educativas.

Mayorga (1999) sostiene que dicho cambio debe responder a las tendencias más relevantes de la época actual, que condicionan el desarrollo de toda sociedad, tales como la democratización, la globalización y la revolución tecnológica mundial.

Los mecanismos para evaluar y certificar fiablemente la calidad de la educación tienen mucha importancia para todos los niveles y modalidades de la misma.

Para Mayorga (1999) la calidad es un concepto multidimensional que se refiere tanto al producto de la educación como a los recursos y procesos que lo producen; tanto al nivel absoluto de sus resultados como a los incrementos generados en el proceso; tanto a elementos cognoscitivos como a elementos éticos del aprendizaje.

También es esencial a la educación de buena calidad la transmisión de valores socialmente consensuados, como pueden ser los asociados a la responsabilidad individual, el respeto a los derechos humanos, la solidaridad y la protección del ambiente, entre otros.

Las universidades al realizar su misión a través de las funciones de docencia, investigación y extensión, concretizan en actividades su razón de ser.

Mediante la docencia, las instituciones transmiten conocimientos, habilidades y valores a los educandos; se preparan los profesionales de todo tipo que requiere cualquier país para su funcionamiento habitual y su desarrollo futuro.

La I+D crea nuevos conocimientos, procesos y productos o modificaciones sustanciales de éstos; la l+D es la concretización del esfuerzo creativo del más alto nivel mental, así como el motor que continuamente alimenta la innovación en los sistemas de producción y las otras funciones universitarias.

Mediante la extensión (llamada a veces "proyección social"), las instituciones de educación superior prestan servicios directos, distintos a los de la I+D y la docencia formal, a personas y entidades. Se trata de una amplia gama de actividades que incluye las asesorías técnicas, los cursos no formales, las publicaciones, el servicio social de los estudiantes, las conferencias y seminarios, los conciertos, las exposiciones de arte y muchas otras.

En la actividad de extensión profundizaremos, por considerarla la menos desarrollada, a pesar de constituir el medio por excelencia a través del cual se pueden dar respuestas a las necesidades y demandas sociales. 
Se considera necesario promover la evaluación del componente extensión ya que de esa manera se contará con la herramienta metodológica, el juicio crítico y las propuestas para su mejoramiento.

Mediante la evaluación se mejorará la calidad institucional a través de interrogarse sobre los resultados, y especialmente sobre las acciones, identificando problemas y comprendiéndolos en su contexto. Se crearán las condiciones óptimas para que los participantes, incluyendo al evaluador, mejoren su comprensión sobre la realidad institucional.

Un primer paso sería crear una unidad de autoevaluación que podría tener los siguientes objetivos:

- Promover la cultura de la autoevaluación como un mecanismo de mejora de la gestión institucional.

- Favorecer el cambio tendiente a la calidad académica.

- Promover el diálogo, la reflexión y el autoanálisis para definir las fortalezas y debilidades en los diferentes aspectos del quehacer institucional.

- Proponer alternativas correctivas a las limitaciones encontradas.

Parte importante del éxito del proceso radica en las cualidades de los componentes de la Unidad. De ahí la importancia de su conformación y del adecuado equilibrio entre sus miembros.

La conducción del proceso exige que tanto el equipo como su coordinador sean técnicamente competentes en el manejo del mismo, en especial en la generación y análisis de información, capacidad de convocatoria y manejo de equipos de trabajo, en la asignación de prioridades y en el equilibrio y exposición de los resultados de la autoevaluación.

Por otro lado, sus funciones serían:
- Dirigir el proceso de autoevaluación institucional;

- Definir y solicitar los recursos y condiciones básicas para el desarrollo del trabajo de la Unidad;

- Definir el mecanismo de trabajo de la Unidad;

- Establecer las funciones de sus miembros;

- Diseñar los instrumentos de evaluación;

- Validar los instrumentos y aplicarlos;

- Procesar, analizar y sintetizar la información obtenida;

- Informar los resultados de la evaluación interna;

- Listar propuestas de solución a los problemas identificados.

Un segundo paso sería la realización de Jornadas de sensibilización hacia la autoevaluación y sus ventajas para la institución en el marco de la Evaluación y acreditación de Programas de Instituciones de Educación Superior del Mercosur.

Se deberá informar por escrito a todos los miembros de la institución sobre el trabajo que realizará la Comisión y sobre lo que se espera de cada uno al proporcionar información y opiniones.

En reuniones con los miembros de cada sector se obtendrán informaciones consultando las fuentes que correspondan a cada caso en particular. La Comisión mantendrá copias de la documentación que respalde las conclusiones a que llegue.

Se han establecido dimensiones y sus componentes a los efectos de la evaluación y que se detallan a continuación:

Dimensión 1: Contexto institucional

Componentes: - Características de las Carreras 
- Organización, administración y gestión de las Carreras

Dimensión 2: Proyecto académico

Componentes: - Plan de estudios

- Enseñanza

- Aprendizaje

- Investigación

- Extensión, vinculación

y cooperación

Dimensión 3: Docentes, alumnos y graduados

Componentes: - Docentes

- Alumnos

- Graduados

Dimensión 4: Recursos humanos e Infraestructura

Componentes: - Edificaciones y su infraestructura

ciones especiales

- Biblioteca

- Laboratorios e instala-

Particularmente nos interesa de la Dimensión PROYECTO ACADÉMICO, el Componente EXTENSIÓN, VINCULACIÓN Y COOPERACIÓN.

Al respecto, en el ámbito del Mercosur y en el proceso de acreditación de las Carreras de Ingeniería, Medicina y Agronomía se definieron criterios e indicadores para la actividad de evaluación.

A continuación se detallan los mismos y que hacen referencia a la extensión universitaria (Quadro 1).

Un siguiente paso sería la elaboración de los instrumentos de recolección de datos, estableciendo las fuentes de información a las que se recurrirán.

Las fuentes de información en la Universidad constituyen: las normativas, documentos institucionales, datos cuantitativos, planes, programas y proyectos, y la opinión de los actores institucionales.

Posteriormente deberá establecerse el tipo de información que se requiere a cada fuente, evaluando las diferentes alternativas para obtenerla, es decir, cuáles son los tipos de instrumentos que pueden emplearse.

Para la obtención de información sobre el componente EXTENSIÓN, VINCULACIÓNY COOPERACIÓN se propone la elaboración de cuestionarios a alumnos, directivos y egresados (Anexo).

El procesamiento de los datos estará a cargo del equipo de Autoevaluación que sistematizará, analizará e interpretará toda la información reunida.

La información obtenida debe ser evaluada en función a los siguientes criterios:

\section{Relevancia}

- Pertinencia

- Impacto

- Adecuación

- Oportunidad

\section{Efectividad}

- Formulación de metas explícitas

- Cumplimiento de metas

\section{Disponibilidad de recursos}

- Humanos

- Materiales

- Información

\section{Eficiencia}

- Administrativa

- Académica

\section{Eficacia}

- Adecuación de recursos

- Relación costo-efectividad

- Relación costo-beneficio

Es conveniente que se compare la información sobre un mismo aspecto obtenida de diferentes fuentes, de manera a tener más elementos de juicio. 
Quadro 1. Dimensión: proyecto académico. Componente: extensión, vinculación y cooperación.

\begin{tabular}{|c|c|c|c|}
\hline Criterios & Indicadores & Fuentes de Información & Instrumentos \\
\hline $\begin{array}{l}\text { Actividades de extensión } \\
\text { cultural: } \\
\text { La Institución y las } \\
\text { Carreras contemplan la } \\
\text { extensión cultural propia y } \\
\text { la transmisión de otras } \\
\text { fuentes culturales a la } \\
\text { comunidad. }\end{array}$ & $\begin{array}{l}\text { Proyección cultural hacia la } \\
\text { comunidad. } \\
\text { Existencia de actividades que } \\
\text { propicien la creatividad, el } \\
\text { desarrollo de ideas, la } \\
\text { transformación tecnológica, la } \\
\text { promoción de talentos, como } \\
\text { propulsor cultural de la } \\
\text { comunidad. }\end{array}$ & $\begin{array}{l}\text { Programas y proyectos de } \\
\text { extensión cultural. } \\
\text { Normativa de la Institución } \\
\text { y las Carreras. }\end{array}$ & $\begin{array}{l}\text { Análisis de la } \\
\text { Normativa. } \\
\text { Entrevistas a } \\
\text { autoridades, } \\
\text { docentes y } \\
\text { alumnos. }\end{array}$ \\
\hline $\begin{array}{l}\text { Cursos de actualización } \\
\text { profesional permanente. } \\
\text { La Institución y las } \\
\text { Carreras contemplan la } \\
\text { extensión de } \\
\text { conocimientos científicos } \\
\text { y profesionales hacia los } \\
\text { graduados o hacia } \\
\text { profesionales de } \\
\text { disciplinas vinculadas. Se } \\
\text { promueve el proceso de } \\
\text { educación continua. Está } \\
\text { dirigida y programada en } \\
\text { función a la demanda de } \\
\text { los graduados y las } \\
\text { necesidades de la región. }\end{array}$ & $\begin{array}{l}\text { Existencia de Cursos de } \\
\text { actualización por áreas de } \\
\text { conocimiento que permitan } \\
\text { acercar a los graduados a las } \\
\text { innovaciones tecnológicas y } \\
\text { científicas. } \\
\text { Existencia de vinculación con } \\
\text { el sector de la producción } \\
\text { para recibir y transferir } \\
\text { conocimientos a alumnos y } \\
\text { graduados. } \\
\text { Existencia de programas de } \\
\text { formación en especialidades, } \\
\text { maestrías o doctorados } \\
\text { vinculados a las Carreras. } \\
\text { Existencia de mecanismos de } \\
\text { comunicación para llegar al } \\
\text { sector involucrado. }\end{array}$ & $\begin{array}{l}\text { Planes y proyectos de } \\
\text { extensión. }\end{array}$ & $\begin{array}{l}\text { Entrevistas a: } \\
\text { - Docentes; } \\
\text { - Graduados; } \\
\text { - Miembros del } \\
\text { sector productivo. }\end{array}$ \\
\hline $\begin{array}{l}\text { Programas de educación a } \\
\text { distancia. } \\
\text { La institución cuenta con } \\
\text { medios de difusión a } \\
\text { distancia que permitan } \\
\text { llevar adelante el proceso } \\
\text { educativo no presencial. }\end{array}$ & $\begin{array}{l}\text { Utilización de técnicas } \\
\text { apropiadas para el proceso de } \\
\text { enseñanza aprendizaje aplica- } \\
\text { do al estudio no presencial. } \\
\text { Existencia de mecanismos de } \\
\text { evaluación, tomado a distan- } \\
\text { cia, que garantice la calidad y } \\
\text { suficiencia del conocimiento } \\
\text { adquirido. }\end{array}$ & $\begin{array}{l}\text { Programas de educación } \\
\text { a distancia disponibles. }\end{array}$ & $\begin{array}{l}\text { Entrevistas a } \\
\text { directivos y } \\
\text { docentes. } \\
\text { Encuesta a } \\
\text { usuarios. }\end{array}$ \\
\hline
\end{tabular}


Conclusão Quadro 1.Dimensión: proyecto académico. Componente: extensión, vinculación y cooperación.

\begin{tabular}{l} 
Criterios \\
\hline Servicios prestados a la \\
comunidad. \\
Las Carreras contemplan \\
la prestación de servicios \\
a la comunidad, a otras \\
instituciones y a los \\
graduados.
\end{tabular}

Convenios de cooperación con el sector público y privado.

La Institución y las Carreras propician la interrelación con el sector socio productivo y los organismos públicos y privados a través de convenios de cooperación que faciliten el aporte de conocimientos aplicados a la problemática regional.

Cursos para capacitación específica.

La Institución y las Carreras proveen cursos para capacitación específica en técnicas, especialidades o disciplinas vinculadas a las Carreras.

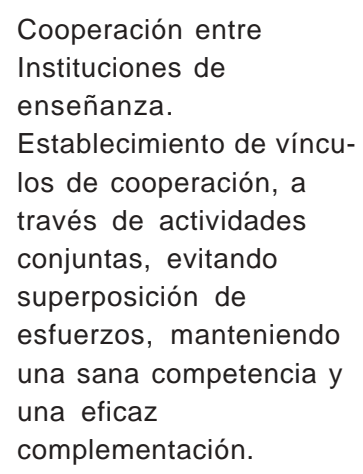

Mecanismos de evaluación de la demanda de cursos específicos.

Existencia de propuestas de capacitación específica, satisfaciendo la demanda de los sectores de la producción y la comunidad.

Existencia de convenios entre las instituciones de enseñanza, investigación o culturales a nivel nacional e internacional. Existencia de mecanismos de medición del impacto de los convenios.

Existencia de una estructura apta para llevar adelante la cooperación.
Fuentes de Información Instrumentos

Planes y proyectos de Entrevistas a servicios de extensión a directivos, la comunidad.

graduados $y$ miembros de la comunidad en las

Materiales disponibles en áreas de los departamentos y especialidad. laboratorios.

Reglamentos de servicios.

Documentación institucional referida al tema de los convenios.

Entrevistas a directivos $y$ encargados del área.

Entrevistas a miembros de los sectores productivos, sociales y organismos públicos y privados vinculados a las Carreras.

Documentación interna y de difusión relativa a los cursos de capacitación específica.

Información sobre la evaluación de la demanda de cursos específicos.

Documentación institucional relativa a la cooperación entre instituciones.

Entrevistas a los miembros de los sectores de la producción vinculados a las disciplinas de las Carreras.

\section{Entrevista a} directivos de la Institución. Verificación de la infraestructura Información sobre la disponible para la evaluación del impacto de extensión. 
Será preciso organizar una discusión de los resultados obtenidos entre los diferentes miembros de la Institución con el fin de lograr un consenso y la apropiación de los resultados por parte de los mismos.

La redacción del Informe de Autoevaluación es la ocasión para que los miembros de la Comisión plasmen el discernimiento reflexivo y valorativo realizado sobre la base de evidencia de la información recolectada a través de los instrumentos de evaluación.

El informe de autoevaluación tendrá una circulación interna en la propia Universidad. El principal destinatario del Informe es la propia unidad académica, objeto de la evaluación.

El segundo destinatario son las autoridades universitarias y el resto de las unidades académicas. Finalmente, los pares y expertos que integren la Comisión de evaluación externa, quienes leerán el referido informe como una primera aproximación a la realidad de la unidad, pero en todo momento tendrán presente que el proceso de autoevaluación puede haber producido modificaciones que el informe no refleje.

Estamos seguros que el proceso de autoevaluación puede reportar grandes beneficios al desarrollo institucional y particularmente a lo que nos interesa en este momento, que es la extensión como interacción entre la universidad y la sociedad.

\section{RE FERE N C I A S}

BELLO DE ARELLANO, M. La educación en Iberoamérica: a través de las declaraciones de las cumbres de jefes de estado y de gobierno y de las conferencias iberoamericanas de educación. Madrid: OEI, 1998. 451p.
En base a todo lo expuesto se propone:

- Fortalecer e innovar la extensión universitaria con el fin de contribuir al desarrollo económico, social y cultural;

- Ampliar la oferta de servicios que ofrecen las universidades para atender a más necesidades y demandas sociales;

- Ampliar la cobertura de sus actividades artísticas y culturales;

- Difundir los conocimientos científicos, tecnológicos y humanísticos;

- Fortalecer el vínculo entre la universidad y la sociedad, para contribuir a solucionar los problemas sociales más urgentes;

- Propiciar una participación activa de docentes, alumnos y graduados en actividades, que dentro de un proceso dinámico y permanente, relacione a la universidad con la sociedad;

- Instaurar la evaluación institucional continua como mecanismo que oriente la planificación y la toma de decisiones.

Finalmente, la extensión deberá promover y gestionar una real y comprometida interacción entre el total del cuerpo universitario y el medio social. Esta interrelación permitirá su inserción en el medio, transfiriendo conocimiento y siendo receptor de la dinámica social y su problemática.

FEMOSO, J. Más allá de la autonomía. París: UNESCO, 1996. 103p.

MAYORGA, R. Los desafíos a la universidad latinoamericana en el siglo XXI Revista Ibero-americana de Educación, n. 21, p.25-40, 1999. 


\section{B I B LIO G RA FíA C O NSULTA D A}

ARGENTINA. Ministerio de Educacion, Ciencia y Tecnologia. Comisión Nacional de Evaluación y Acreditación Universitaria. Lineamientos para la Evaluación Institucional. Disponible em: $<$ www.coneau.edu.ar/docs-varios>.

BRÍTEZ SÁNCHEZ, S.S. et al. Las universidades paraguayas y su proyección a la sociedad. San Lorenzo: Carrera de Bibliotecología de la Facultad Politécnica, 2002. 92p.

CEPEDA LÓPEZ, G. et al. Fundamentos teóricos y práctica de la autoevaluación de programas académicos en la educación superior. Santafé de Bogotá: CEJA, 1999. 159p.

GANDOLFO, D.M.; SUÑE, L. (Coord.). Sistema experimental de evaluación de carreras de
Ingeniería: instrumento para validación del sistema Santiago de Chile, 2001.

GARDUÑO ESTRADA, L.R. Hacia un modelo de evaluación de la calidad de instituciones de educación superior. Revista Iberoamericana de Educación, n.21, p.93-103, 1999.

NEVO, D. Evaluación basada en el centro: un diálogo para la mejora educativa. [S.I.]: Ediciones Mensajero, 1997. 207p.

RODRÍGUEZ ALONSO, R.A. Instrumentos para la autoevaluación de instituciones educativas. Santiago de Chile: Santillana, 2001.175p.

Ruíz Ruíz, J.M. Cómo hacer una evaluación de centros educativos. Madrid: Narcea, 19_.. 


\section{ANEXO \\ UNIVERSIDAD NACIONALDE ASUNCIÓN \\ FACULTAD POLITÉCNICA}

\section{Unidad de Autoevaluación Institucional}

\section{Cuestionario para Autoridades}

En el marco del proceso de autoevaluación su participación es de suma importancia.

Nos interesa conocer la valoración que adjudica a los proyectos de Extensión, Vinculación y Cooperación de nuestra Facultad.

Gracias por contestar las preguntas del siguiente cuestionario.

1. Tiene la Facultad Politécnica una política establecida con respecto a:
a- La extensión
b- La vinculación
c- La cooperación

Sí

Sí

Sí

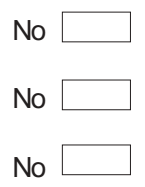

2. Las actividades de Extensión que se realizan son pertinentes
a- A las exigencias de las materias
b- A las líneas de investigación de la institución
c- Para las competencias establecidas en el perfil del egresado
d- A las necesidades de la comunidad
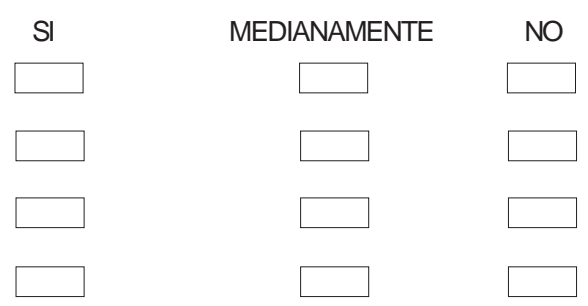

3. Cuál de las siguientes actividades se desarrollan regularmente:

a) Actividades culturales

b) Cursos de actualización profesional para graduados

c) Cursos de educación a distancia en las diferentes carreras ofrecidas

d) Servicios técnico-profesionales a las empresas

e) Firma de convenios con otras instituciones relacionadas a nivel nacional a nivel internacional

f) Cooperación con otras instituciones de enseñanza afines 
4. Las actividades de Extensión que se realizan reportan beneficios para:

a) Profundizar los contenidos

b) Relacionar la teoría con la práctica

c) Desarrollar la sensibilidad social de los futuros profesionales

d) Retroalimentar con información de la sociedad los Planes de estudio

5. Para la prestación de los servicios de Extensión cuenta la Facultad con:

a) La Infraestructura necesaria

b) El personal idóneo para la coordinación de las actividades

6. Las actividades de extensión son realizadas por:

a) Los alumnos solamente

b) Los profesores solamente

c) Los alumnos y los profesores

7. Existen mecanismos de evaluación del impacto de los convenios de cooperación firmados?

$\mathrm{SI}$

NO

Si su respuesta es positiva, mencione cuáles son los mecanismos de evaluación 


\section{UNIVERSIDAD NACIONALDE ASUNCIÓN FACULTAD POLITÉCNICA}

\section{Unidad de Autoevaluación Institucional \\ Cuestionario para Estudantes}

En el marco del proceso de autoevaluación su participación es de suma importancia.

Nos interesa conocer la valoración que adjudica a los proyectos de Extensión, Vinculación y Cooperación de nuestra Facultad.

Gracias por contestar las preguntas del siguiente cuestionario.

No necesita identificarse. El cuestionario es anónimo.

1. Participan los estudiantes en las actividades de extensión?
Sí
De qué forma?

No

2. Cuáles son las actividades desarrolladas hasta la fecha?

Servicios sociales a la comunidad

Pasantías

Cursos de actualización

Actividades culturales

Firma de convenios interinstitucionales

Otros

3. Qué podría hacerse para mejorar la participación de los estudiantes?

4. Considera que las actividades de extensión contribuyen a la formación académica?

Sí

No
De qué manera? 


\section{UNIVERSIDAD NACIONAL DE ASUNCIÓN \\ FACULTAD POLITÉCNICA}

\section{Unidad de Autoevaluación Institucional}

\section{Cuestionario para Graduados}

En el marco del proceso de autoevaluación su participación es de suma importancia.

Nos interesa conocer la valoración que adjudica a los proyectos de Extensión, Vinculación y Cooperación de nuestra Facultad.

Gracias por contestar las preguntas del siguiente cuestionario.

No necesita identificarse. El cuestionario es anónimo.

1. Tiene conocimiento de las ofertas de cursos de actualización profesional ofrecidos por la Facultad Politécnica?

Sí

No

2. Ha participado de dichos cursos?

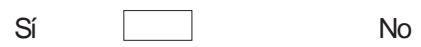

3. Cuántos de ellos tuvieron relación con su especialidad?
Todos
Mayoría
Ninguno

4. Responden a sus expectativas las temáticas y enfoques abordados en los cursos?

Todos Mayoría $\square \quad$ Ninguno

5. Que cree conveniente hacer para mejorar los mecanismos de relacionamiento de la Facultad con sus egresados? 